\section{DECIPHERING THE MECHANISMS OF DEVELOPMENTAL DISORDERS (DMDD): A POWERFUL NEW RESOURCE FOR STUDYING CONGENITAL HEART DISEASE}

Tim Mohun*. The Francis Crick Institute

\subsection{6/heartjnl-2017-311726.175}

Identifying the genetic components of heart disease remains challenging despite the transformation of clinical genetics by new DNA sequencing technologies. For congenital heart disease (CHD), animal models have provided a fruitful way to identify genes necessary for differentiation of cardiac tissue and subsequent morphogenesis of the embryo heart. These have identified candidate determinants of CHD, and their genetic manipulation in animal models such as the mouse offers the opportunity to identify the molecular mechanisms whose disruption results in foetal cardiac abnormalities. This is of potential clinical value not only for diagnostic screening of CHD; it also identifies critical molecular pathways whose disruption by environmental factors may account for the $80 \%$ of CHD for which there is no attributable genetic cause.

"Deciphering the Mechanisms of Developmental Disorders" (DMDD) is a five-year study dedicated to identifying genes essential for embryo development, many of which affect heart development when targeted by selective gene knockout. Here we present the first systematic study of the range, severity and penetrance of defects affecting the heart by the end of organogenesis (E14.5) in such embryonic lethal mouse mutants. Using comprehensive $3 \mathrm{D}$ imaging at near histological resolution, we have examined embryos from 42 different gene knockouts, identifying morphological abnormalities affecting the heart and great vessels. Abnormalities were scored using the Mouse Phenotype (MP) Ontology and all image and phenotype data from the study is freely available to the scientific community at dmdd.org.uk.

Cardiac defects were detected in 33 of the first 42 mutant lines studied (78\%) and many comprise genes studied either for the first time or not previously associated with cardiac abnormalities. Many of the defects identified match congenital defects identified in newborn babies. Individual mutant lines varied widely in the number of unique cardiac phenotypes they exhibited, with as many as 20 distinct types in the most severely affected embryos. Over half of all lines examined showed defects affecting the atrial or ventricular septum; one third of the lines had abnormalities affecting development of the outflow tract (overriding aorta, double outlet right ventricle, persistent arterial trunk, transposition of the great arteries); a quarter showed defects affecting the structure of one or more heart chambers and a fifth of the lines had abnormalities in the atrio-ventricular junction.
These results demonstrate the remarkable power of an unbiased genetic screen in the mouse to reveal novel CHD genes when simply filtered by embryonic lethality. The DMDD data offers a rich and expanding resource for further study of both normal mammalian heart development and the aetiology of congenital heart disease.

\section{APPLICATION OF CARDIAC MRI TO QUANTITATIVELY ASSESS MYOCARDIAL DAMAGE IN ISOPROTERENOL- INDUCED HEART FAILURE}

${ }^{1}$ Xiao Xiao Han*, ${ }^{2}$ Georgina Ellison, ${ }^{1}$ Mark F Lythgoe, 'Daniel J Stuckey. ${ }^{1}$ UCL Centre for Advanced Biomedical Imaging, University College London; ${ }^{2}$ Centre of Human and Aerospace Physiological Sciences, King's College London

\subsection{6/heartjnl-2017-311726.176}

Introduction Cardiovascular disease is the Number-1 cause-ofdeath globally with an estimation of 17.5 million deaths annually. Being one of the most active organs of the body, the heart is vulnerable to damage. Isoproterenol (ISO) injection into mice can induce a stress cardiomyopathy model similar to Takotsubo cardiomyopathy. Cardiac MRI permits the detection, visualisation, quantification, and progression-monitoring of cardiac defects non-invasively, making it useful for monitoring stress cardiomyopathy. In this study, we used MRI to study the effects of ISO injection on cardiac structure and function in both immune-competent and immune-deficient mice.

Methods and Materials 12 week old C57B6 $(n=14)$ and immunocompromised NOD-SCID mice $(n=18)$ were assigned to Control and ISO Group with $0.9 \%$ saline or $1 \mathrm{mg} / \mathrm{kg}$ ISO administered daily for 7 days by i.p. injection. At 7 days imaging was performed using a 9.4T MRI system and $38 \mathrm{~mm}$ quadrature birdcage RF coil. Cardiac and respiratory gated cine-MRI was performed in the true short-axis orientation and covered the whole left ventricle. LGE-MRI was performed $20 \mathrm{~min}$ after i.p. injection of $0.5 \mathrm{mmol} / \mathrm{kg}$ Gd-DTPA-BMA using a multi-slice inversion recovery sequence. Data were analysed in a blinded fashion using ImageJ. LGE-MRI images were thresholded to the full width at half maximum of enhanced regions to identify areas of signal enhancement.

\begin{tabular}{lllll} 
Abstract 178 Table 1 & & & & \\
\hline Cardiac Parameters & Control & Is0 & Difference & P value \\
\hline End Diastolic Volume (11/4l) & $52 \pm 2$ & $63 \pm 4$ & 10 & 0.02 \\
End Systolic Volume (i $1 / 4$ l) & $12 \pm 2$ & $23 \pm 4$ & 10 & 0.01 \\
Ejection Fraction (\%) & $77 \pm 2$ & $65 \pm 4$ & -11 & 0.005 \\
Left Ventricular Mass (mg) & $79 \pm 1$ & $92 \pm 7$ & -13 & $\mathrm{~ns}$ \\
\hline
\end{tabular}

Pattern of Fibrosis Distribution

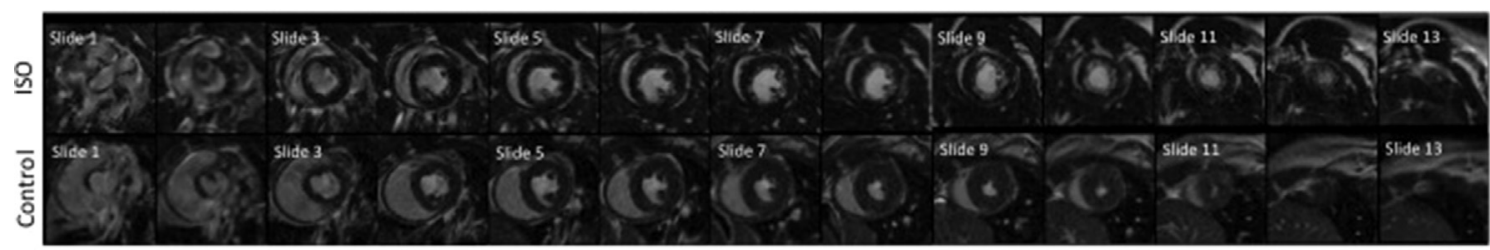

Abstract 178 Figure 1 Late-gadolinium MRI image series (showing all slices from the base to the apex of the heart) in short axis view, with areas of enhancement (apical subendocardial enhancement) corresponding to regions of cardiac fibrosis, thereby demonstrating the endomyocardial nature of the cardiac fibrosis. Above - ISO. Below - Control. 


\begin{tabular}{lllll} 
Abstract 178 & Table 2 & & \\
\hline $\begin{array}{l}\text { Cardiac Morphology } \\
\text { and Fibrosis }\end{array}$ & Control & Iso & \\
\cline { 2 - 5 } & & Immunocompetent & Immunocompromised & $\mathbf{P}$ \\
& & & $35 \pm 5$ & value \\
\hline Fibrotic Mass (mg) & 0 & $16 \pm 1$ & & 0.02 \\
\hline
\end{tabular}

Results ISO caused changes in: 1) cardiac function - increasing end diastolic and end systolic volumes while decreasing ejection fraction, 2) cardiac morphology - increasing the left ventricular mass resulting in hypertrophy (enlargement of the heart), and 3) cardiac fibrosis - apex of the heart being the most vulnerable. A differential-distribution of fibrosis-severity across the heart has also been detected.

Discussion In agreement with initial hypothesis, there was a differed-response between Immunocompetent and Immunocompromised subgroups: the Immunocompetent showed a trend towards cardiac hypertrophy, whereas the Immunocompromised were more vulnerable to fibrosis. This cardioprotective role of the immune system could be due to CXCR4 signalling that is also implicated in cancer-metastasis, stem-cellmigration, angiogenesis, and haematopoiesis, which we will follow up.

Conclusion ISO-murine-disease-model has been established and characterised to study myocardial damage, with cardiac function, morphology, and damage in the form of fibrosis detected, visualised, and quantitatively assessed using cardiac MRI. ISO caused changes in cardiac function, morphology, and fibrosis. There was also a differential-distribution of fibrosis-severity across the heart as shown visually by high-resolution sequential MRI images.

\section{PHOSPHODIESTERASE-5 INHIBITION WITH SILDENAFIL SUPPRESSES CALCIUM WAVES BY REDUCING SARCOPLASMIC RETICULUM CONTENT}

David Hutchings*, Katharine Dibb, Charles Pearman, George Madders, Lori Woods, David Eisner, Andrew Trafford. University of Manchester

10.1136/heartjnl-2017-311726.177

Rationale: Occurrence of diastolic $\mathrm{Ca}^{2+}$ waves in cardiac myocytes leads to arrhythmias by inducing delayed after-depolarisations. Waves are initiated when sarcoplasmic reticulum (SR) content reaches a critical threshold level. The phosphodiesterase-5 inhibitor sildenafil (Sil) is antiarrhythmic in mammalian myocardial ischaemia models, while Sil reduces $\mathrm{Ca}^{2+}$ transient amplitude and sarcoplasmic reticulum (SR) $\mathrm{Ca}^{2+}$ content in rat myocytes.

Objective: To determine effects of Sil on propensity to $\mathrm{Ca}^{2+}$ waves in the large mammal.

Methods: Sheep ventricular myocytes were voltage clamped and intracellular $\mathrm{Ca}^{2+}$ measured using Fura-2. Cells were paced at $0.5 \mathrm{~Hz}$ with depolarisations from $-40 \mathrm{mV}$ to $+10 \mathrm{mV}$. When at steady state, waves were induced with 10$15 \mathrm{mM} \mathrm{Ca}^{2+}$. Upon regular waving, Sil $(1 \mu \mathrm{M})$ was applied. To determine threshold SR content, caffeine $(10 \mathrm{mM})$ was added immediately following a wave, and both wave and caffeine-induced $I_{\mathrm{NCX}}$ integrated. Differences between groups were determined using students paired t tests.
Results: Increasing external $\mathrm{Ca}^{2+}$ to $10-15 \mathrm{mM}$ increased SR content and induced diastolic waves. Sildenafil abolished waves in 9/15 cells. In cells where Sil terminated waves, SR content was reduced below threshold. In addition, Sil treatment was associated with a reduced rate constant of SERCA ( $\mathrm{k}_{\text {SERCA }}-66.0 \pm 9.9 \%$ of control, $\mathrm{p}<0.005$ ), an initial (first $4 \mathrm{~s}$ ) increase in sarcolemmal efflux via the $I_{\mathrm{NCX}}$ tail current $(+142$ $\pm 36.4 \%, \mathrm{p}<0.01)$, and reduced sarcolemmal influx via $I_{\mathrm{Ca}-\mathrm{L}}$ $(-30.5 \pm 5.6 \%, \mathrm{p}<0.005)$. In cells continuing to wave in Sil, SR threshold for waves was unchanged $\left(126.9 \mu \mathrm{molL}^{-1}\right.$ ctrl vs 147.2 $\left.\mathrm{molL}^{-1} \mathrm{Sil}, \mathrm{p}=0.6\right)$. In unstimulated cells spontaneously waving in $10-15 \mathrm{mM} \mathrm{Ca}^{2}$, sildenafil reduced wave frequency (6.3 waves per $20 \mathrm{~s}$ vs $2.7, \mathrm{p}<0.005)$. The protective effect of sildenafil on both wave models was abolished when cells were pre-incubated with the PKG inhibitor, KT5823. Sildenafil suppression of waves was also observed in cells from animals in end-stage heart failure, while Sil suppressed ventricular ectopy and episodes of torsades de pointes in vivo in a sheep model of LQT2.

Conclusions: Sildenafil suppresses waves induced by elevated external $\mathrm{Ca}^{2+}$ via a PKG-dependent mechanism, and mediated by a reduction in SR content, which itself is caused by reduced SERCA function \pm reduced $I_{\text {Ca-L }}$. These findings highlight novel antiarrhythmic properties of PDE5 inhibition and translate to suppression of triggered arrhythmias in vivo.

Funding: British Heart Foundation.

\section{THE AORTA CAN ACT AS SITE OF T CELL PRIMING AND PROMOTES A LOCAL CD4+ ADAPTIVE IMMUNE RESPONSE IN EARLY STAGE ATHEROSCLEROSIS IN APOLIPOPROTEIN-E-/- MICE}

Neil MacRitchie*, Gianluca Grassia, Catherine Hughes, Jonathan Noonan, Juliane Schroeder, Paul Garside, Pasquale Maffia. University of Glasgow

10.1136/heartjnl-2017-311726.178

Artery tertiary lymphoid organs (ATLOs) in the adventitia adjacent to intimal plaque in aortas from aged apolipoprotein$\mathrm{E}(\mathrm{apoE})^{-/-}$mice have recently been shown by us to orchestrate the aortic immune response in the advanced stages of experimental atherosclerosis, highlighting the importance of the vascular immune response and its contribution to the pathology. Antigen presenting cells (APCs) and $\mathrm{T}$ cells are found in both human and animal na $\tilde{A} f \hat{A}^{-}$ve and atherosclerotic vessels; however, the mechanisms leading to $\mathrm{T}$ cell activation in the arterial wall remain poorly understood. Here we utilised flow cytometry to present a quantitative assessment of the major antigen presenting cells in both $\mathrm{C} 57 \mathrm{BL} / 6$ wild type (WT) and apoE ${ }^{-/-}$mice in the aorta, aortic draining lymph nodes and spleen. By employing a model antigen and antibody detections system (E $\alpha-G F P / Y-A e)$, it was possible to assess the ability of aortic APC subsets to present antigen in vivo. We also performed a comprehensive phenotypic analysis of $\mathrm{CD}^{+}$ $\mathrm{T}$ cells in healthy versus atherosclerotic aorta using surface marker expression and cytokine signatures. This study revealed that aortas from atherosclerotic mice contained more $\mathrm{CD} 4^{+} \mathrm{T}$ cells and more Th1 T cells (IFN- $\gamma^{3}+$ ) compared to WT, as well as showing a phenotypic switch from a nave to an activated phenotype. On the contrary, changes in $\mathrm{T}$ cell phenotype in the spleen and draining lymph nodes between WT and apoE $^{-/}$mice were either equivalent or modest. One hypothesis regarding the presence of $\mathrm{T}$ cell autoreactive clones 